\title{
Monitoring community mobilisation and organisational capacity among high-risk groups in a large-scale HIV prevention programme in India: selected findings using a Community Ownership and Preparedness Index
}

\author{
Pradeep Narayanan, ${ }^{1} \mathrm{~K}$ Moulasha, ${ }^{1}$ Tisha Wheeler, ${ }^{2}$ James Baer, ${ }^{3}$ \\ Sowmyaa Bharadwaj, ${ }^{1}$ T V Ramanathan, ${ }^{4}$ Tom Thomas ${ }^{1}$
}

${ }^{1}$ Praxis Institute for Participatory Practices, New Delhi, India ${ }^{2}$ Futures Group Washington DC, USA (formerly with the Bill and Melinda Gates Foundation, India Office, New Delhi, India) Independent Consultant, London, UK

${ }^{4}$ Department of Statistics, University of Pune, Pune, India

\section{Correspondence to}

Pradeep Narayanan, Research and Consultancies, Praxis Institute for Participatory Practices, C - 75, South Extension, Part II, New Delhi-110049, India; pradeepn@praxisindia.org

Accepted 25 May 2012 Published Online First 5 July 2012

\section{UNLOCKA}

This paper is freely available online under the BMJ Journals unlocked scheme, see http:// jech.bmj.com/site/about/ unlocked.xhtml

\section{ABSTRACT}

Background In a participatory approach to health and development interventions, defining and measuring community mobilisation is important, but it is challenging to do this effectively, especially at scale.

Methods A cross-sectional, participatory monitoring tool was administered in 2008-2009 and 2009-2010 across a representative sample of 25 community-based groups (CBGs) formed under the Avahan India AIDS Initiative, to assess their progress in mobilisation, and to inform efforts to strengthen the groups and make them sustainable. The survey used a weighted index to capture both qualitative and quantitative data in numeric form. The index permitted broad, as well as highly detailed, analysis of community mobilisation, relevant at the level of individual groups, as well as state-wide and across the whole programme.

Results The survey demonstrated that leadership and programme management were the strongest areas among the CBGs, confirming the programme's investment in these areas. Discussion of the Round 1 results led to efforts to strengthen governance and democratic decision making in the groups, and progress was reflected in the Round 2 survey results. CBG engagement with state authorities to gain rights and entitlements and securing the long-term financial stability of groups remain a challenge.

Conclusion The survey has proven useful for informing the managers of programmes about what is happening on the ground, and it has opened spaces for discussion within community groups about the nature of leadership, decision making and their goals, which is leading to accelerated progress. The tool provided useful data to manage community mobilisation in Avahan.

\section{INTRODUCTION}

In India, as in other countries, community mobilisation has proved valuable in working with sex workers and other groups at high risk of HIV infection, as an approach that can improve HIV risk reduction and enhance programme relevance and sustainability. ${ }^{1-6}$ However, attention to the operational approaches necessary to achieve these goals is often limited to local management rather than following a broader data-informed strategy. In instances where community mobilisation activities have integrated a measurement approach, best prac- tice has demonstrated that the communities being assessed should be involved in the design of the assessment, so as to ensure that results are legitimate and immediately beneficial to the communities engaged. ${ }^{7}$ Approaches that succeed in involving communities, however, tend to operate at a small scale, and measurements tend to be context-specific.

There are a few precedents for measuring community mobilisation in large-scale interventions. ${ }^{8}$ The Avahan India AIDS Initiative, a 10-year programme to reduce HIV transmission in six highprevalence states, which has a significant component of community mobilisation, is attempting to do this. ${ }^{9} 10$ The communities referred to by the programme are formed from the 320000 female sex workers (FSWs), high-risk men who have sex with men (HR-MSM), transgendered people (TGs) and injecting drug users (IDUs) for whom the programme operates. $^{9}$

For Avahan, the challenge of community mobilisation has been threefold: to develop cohesion and a sense of shared identity among marginalised individuals; to build their capacity to oversee and manage aspects of HIV prevention programming; and to empower them to define and implement their own agendas for change as a community. ${ }^{8}$

Avahan interventions include behaviour change communication, condom and needle/syringe distribution, provision of sexually transmitted infection treatment and links to HIV care services. Community mobilisation is an integrated component of the programme and has been implemented in several stages, beginning with the programme interacting primarily with paid peer outreach workers delivering services, and progressing to the strengthening of community-based groups (CBGs), which, over time, will define their own agendas and become pressure groups advocating for services, rights and entitlements for their communities. ${ }^{8} 11$

When the Avahan Initiative ends in 2013, responsibility for managing service delivery will transition from Avahan to the Indian Government, while responsibility for advocacy, crisis response (addressing violence), access to entitlements and organisational development will shift to the CBGs. ${ }^{12}$ Since 2009, Avahan's community mobilisation efforts have focused on making CBGs 'transition ready' by strengthening their organisational capacities, so that they can hold the government 
accountable for programme quality, manage interventions related to vulnerability reduction, pursue broader, self-defined agendas and sustain themselves beyond the lifetime of Avahan. ${ }^{13}$

In order to monitor the progress of community mobilisation among the CBGs formed under Avahan, to assess their 'transition readiness' and to provide data for management and planning, annual monitoring has been conducted since 2009 using a tool designed for these purposes. ${ }^{10}$ This paper presents findings from 25 CBGs of FSWs and HR-MSM that were assessed in two rounds of monitoring, conducted in 2008-2009 and 2009-2010, to illustrate how the tool can be used to assess the performance of individual CBGs, and to give an overview of how Avahan used the data to monitor the progress of community mobilisation at the broader programme level.

\section{METHODS}

\section{Conceptual framework}

A tool for prospective monitoring of CBGs, the Community Ownership and Preparedness Index (COPI), was designed, combining a cross-sectional survey approach with a participatory monitoring approach. ${ }^{14}$ The conceptualisation and design of the COPI are described in detail in an accompanying paper and are summarised here. ${ }^{10}$ The COPI drew upon a review of relevant literature and discussions with high-risk community members and professionals working in the fields of community development and HIV prevention, in order to delineate power relations among stakeholders (such as CBGs, NGOs, government and the community), and to define and identify the characteristics of a strong CBG. ${ }^{16715-19}$ This process led to the articulation of four overarching dimensions of a CBG's capacity considered essential to transition readiness (table 1). After further discussion with community members and experts, these four dimensions were subdivided into eight components or 'parameters' characteristic of a strong participatory CBG, addressing both its internal functioning and external elements that affect its organisational development (table 1).

Each parameter was comprised of between two and five indicators (23 in total), which were monitored through a set of detailed questions about the CBG's activities, posed to CBG leaders and members, and to the staff of the NGO implementing the intervention (the implementing NGO). The response to each question was given a numeric score, reflecting not only whether an activity took place, but also the level of involvement of CBG leaders and members in planning and executing the activity, reflecting the organisational strength of the $\mathrm{CBG}$ in that particular area. The aggregate score for each indicator was then weighted to reflect the indicator's relative importance to community mobilisation and transition readiness. These weights were determined as part of the COPI design through a process of consultation with community mobilisation experts and community members. The weighted scores for each indi-

Table 1 Dimensions and parameters of Community Ownership and Preparedness Index (COPI) tool

\begin{tabular}{ll}
\hline Dimensions of organisational capacity & Parameters \\
\hline 1. Organisational strength & 1. Leadership \\
& 2. Governance \\
& 3. Decision making \\
2. Sustainability & 4. Resource mobilisation \\
& 5. Networking \\
3. Programme management & 6. Programme management \\
4. Engagement on issues of rights, & 7. Engagement with state to secure rights \\
entitlements and stigma reduction & and entitlements \\
& 8. Engagement with wider society to \\
& reduce stigma \\
\hline
\end{tabular}

cator were totalled to give a parameter score (expressed as a percentage of the maximum score possible). ${ }^{10}$

\section{District choice}

The primary unit of data collection for each CBG for the COPI was the district, which in India is the main administrative unit responsible for provision of HIV/AIDS programming and social entitlements. ${ }^{20}$ In addition, most survey and monitoring activities by both, Avahan and the Government of India, have taken place at this level. ${ }^{20}$ From the point of view of community mobilisation in Avahan, the district generally corresponds to the area of influence for the implementing NGO, and in most cases, for the CBG also.

Given limited resources, the COPI was implemented in a sample of 35 CBGs in 32 of the 83 districts where Avahan was working. In the second round of monitoring, $75 \%$ of these CBGs were retained in the sample for cohort analysis, while the remaining $25 \%$ of the sample consisted of districts that had not been included in the first round. In order to avoid bias in selection of districts to be monitored, stratified random sampling was used to ensure representation of each state where the programme was operating, each state-level partner implementing the programme (large NGOs who sub-granted to local implementing NGOs and provided technical support) and all high-risk groups (FSWs, HR-MSM/TGs and IDUs) covered by Avahan in each state. In the first round of monitoring only, the sampling also insured inclusion of districts where Avahan was the sole HIV prevention intervention ('solo' districts), and those where it was not ('shared' districts). ${ }^{21}$

\section{Implementation}

The first round of the survey was conducted from August 2009 to October 2009, collecting data for the period July 2008-June 2009. The second round of the survey, conducted from August 2010 to October 2010, gathered data for the period July 2009 to June 2010. In each round, the selected CBGs were invited to participate voluntarily, and all consented to do so. Data were collected simultaneously in all states by 25 trained field workers. Working in teams of two, the field workers conducted 5-day visits to each $\mathrm{CBG}$, during which questionnaires were completed through faceto-face interviews with four groups of stakeholders: the CBG's leadership team; committee members; staff of the implementing $\mathrm{NGO}$; and members of the high-risk community.

At the end of each visit, a day was dedicated to presenting the CBG and NGO with a preliminary analysis of data. This immediate feedback fostered active discussion about the monitoring process, the results and possible next steps. A more extensive written report was subsequently provided to the NGO and the $\mathrm{CBG}$, translated into the local language where necessary.

\section{Data analysis}

A database was developed using Filemaker Pro@ ${ }^{22}$ for concurrent input of data (scores) from individual CBGs. Coordinators at the state and national levels checked the completeness and consistency of data collection and entry. Data were analysed by individual and aggregated indicators and parameters, and by variable (state, type of high-risk group, legal registration status of CBG). Progressively higher scores reflected: (a) transition in agency, with the lead role in activities moving from NGO staff to peer educators to community leaders; (b) transition of the community leadership's role, from beneficiary of services to programme implementer to strategic decision maker and (c) transition of the community's capacity to engage with the state and wider society, from being invisible to being assertive. 
Table 2 Community-based groups of FSWs and HR-MSM common to first two rounds of monitoring, by state and date of formation

\begin{tabular}{|c|c|c|c|c|}
\hline \multirow[b]{2}{*}{ State } & \multicolumn{2}{|c|}{ FSW community-based groups } & \multicolumn{2}{|c|}{$\begin{array}{l}\text { HR-MSM } \\
\text { community-based groups }\end{array}$} \\
\hline & $\begin{array}{l}\text { Groups formed } \\
\text { in or before } \\
2007^{*}\end{array}$ & $\begin{array}{l}\text { Groups formed } \\
\text { in or after } \\
2008\end{array}$ & $\begin{array}{l}\text { Groups formed } \\
\text { in or before } \\
2007^{*}\end{array}$ & $\begin{array}{l}\text { Groups formed } \\
\text { in or after } \\
2008\end{array}$ \\
\hline Andhra Pradesh & 2 & 2 & 2 & 2 \\
\hline Karnataka & 3 & 0 & 1 & 0 \\
\hline Maharashtra & 0 & 4 & 1 & 2 \\
\hline Manipur & 0 & 0 & 1 & 0 \\
\hline Nagaland & 1 & 0 & 0 & 0 \\
\hline Tamil Nadu & 2 & 0 & 2 & 0 \\
\hline Total & 8 & 6 & 7 & 4 \\
\hline
\end{tabular}

*Date of formation is defined as the year when members of the CBG first met and agreed to work together as a group. The date of official registration as a community-based organisation (CBO), if this has occurred, may be later. Avahan phase 2 began in 2008, when community mobilisation received substantial focus.

\section{RESULTS}

Selected results from 25 CBGs of FSWs and HR-MSM that were common to the first two rounds of the survey (see table 2) are presented here.

\section{CBO-level analysis}

As part of regular monitoring, results were produced for individual CBGs, as well as for state-level implementing partners. The COPI enables a disaggregation of results at parameter and indicator levels, allowing CBGs and implementing partners to understand where to focus efforts and resources. Figure 1 presents an example of a quantified analysis by parameter for an individual CBG. The parameters of Leadership, Resource Mobilisation and Programme Management were the three strongest parameters for this CBG in both monitoring rounds. The performance of the CBG improved over the two rounds in all parameters except Networking, with the greatest increase (21 percentage points) in Decision Making, and increases of 10-12 percentage points in Resource Mobilisation, Programme Management, Engagement with the State and Engagement with Wider Society.

\section{Programme-level analysis}

Scores obtained on each parameter by the $25 \mathrm{CBGs}$ were aggregated for assessment at the programme level. Figure 2 presents the aggregated median scores over the two rounds of the survey, with the line showing the highest score obtained by any CBG for that parameter. Leadership and Programme Management were the strongest parameters across the programme in both rounds of the survey (ranging from 33\% to $37 \%$ ). Between the two rounds, the programme showed most progress in facilitating systems of Governance (from 23\% to $30 \%$ ) and Engagement with the State (from 17\% to $26 \%$ ). Capacities were also strengthened in the domains of Decision Making and Engagement with Wider Society.

\section{Analysis by high-risk group}

Disaggregating the CBGs by high-risk group (figure 3) reveals differences in the average performance of the groups in various parameters. In the first monitoring exercise, FSW CBGs had higher parameter scores on average than HR-MSM CBGs in four parameters, with the most marked contrast in Resource Mobilisation (a difference of eight percentage points). HR-MSM CBGs had higher parameter scores in three parameters, with the most marked contrast in Programme Management (six percentage points). In Round 2 of the monitoring exercise, the contrasts between the two types of CBG were more strongly drawn: of the five parameters in which FSW CBGs scored higher on average, there was a difference of at least eight percentage points in three parameters-Resource Mobilisation, Programme Management and Engagement with the State-with the difference as high as 15 percentage points in the last of these.

\section{Analysis by age of CBG}

Figure 4 shows the median performance of the CBGs across the two rounds of monitoring, disaggregated by those formed in or before 2007 (15 CBGs), and those formed in or after 2008 (10 CBGs). In the first round of the survey, the older CBGs performed better than the newer ones in all parameters except Engagement with the State and Engagement with Wider Society. The difference was particularly pronounced in Resource Mobilisation, Networking and Programme Management. By the
Figure 1 Parameter scores of a sample community-based group in two rounds of monitoring with Community Ownership and Preparedness Index (COPI) tool.

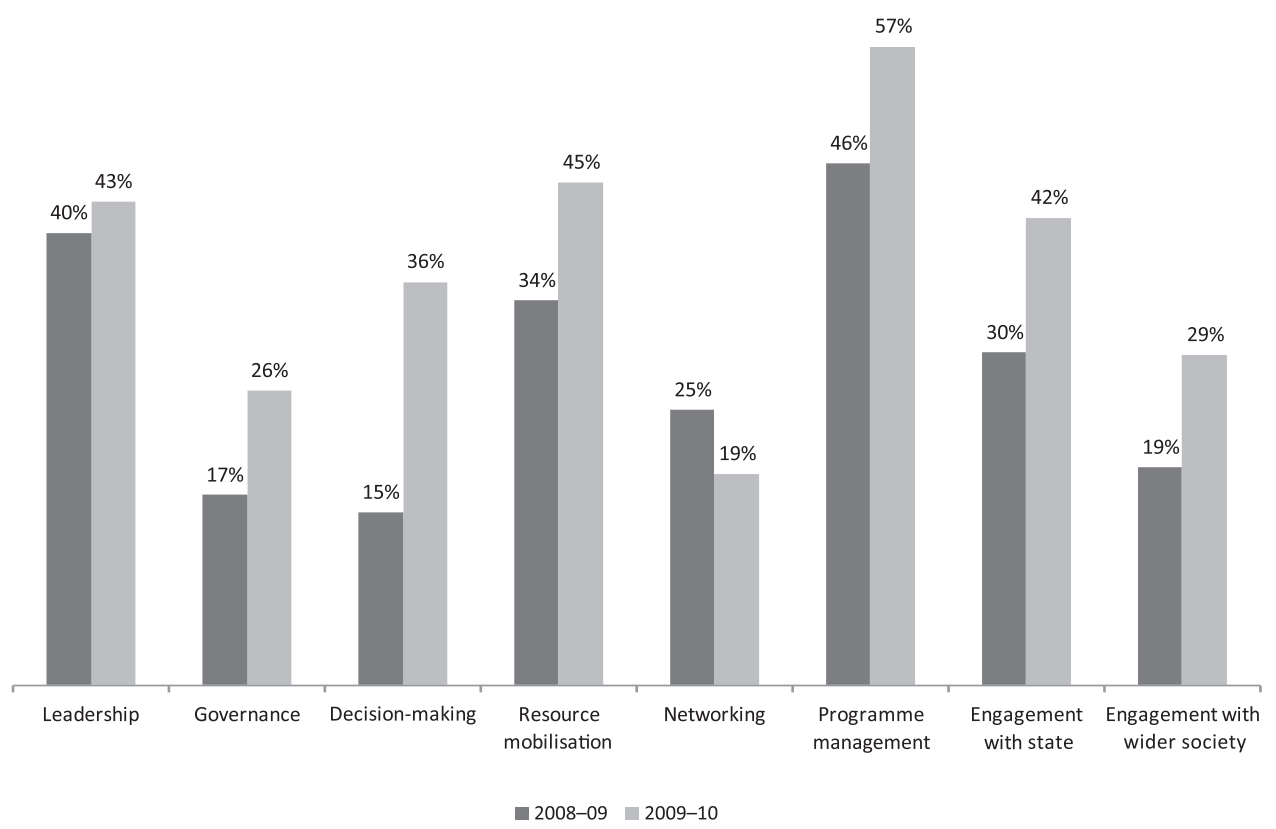


Figure 2 Median and highest parameter scores of 25 communitybased groups in two rounds of monitoring with Community Ownership and Preparedness Index (COPI) tool.

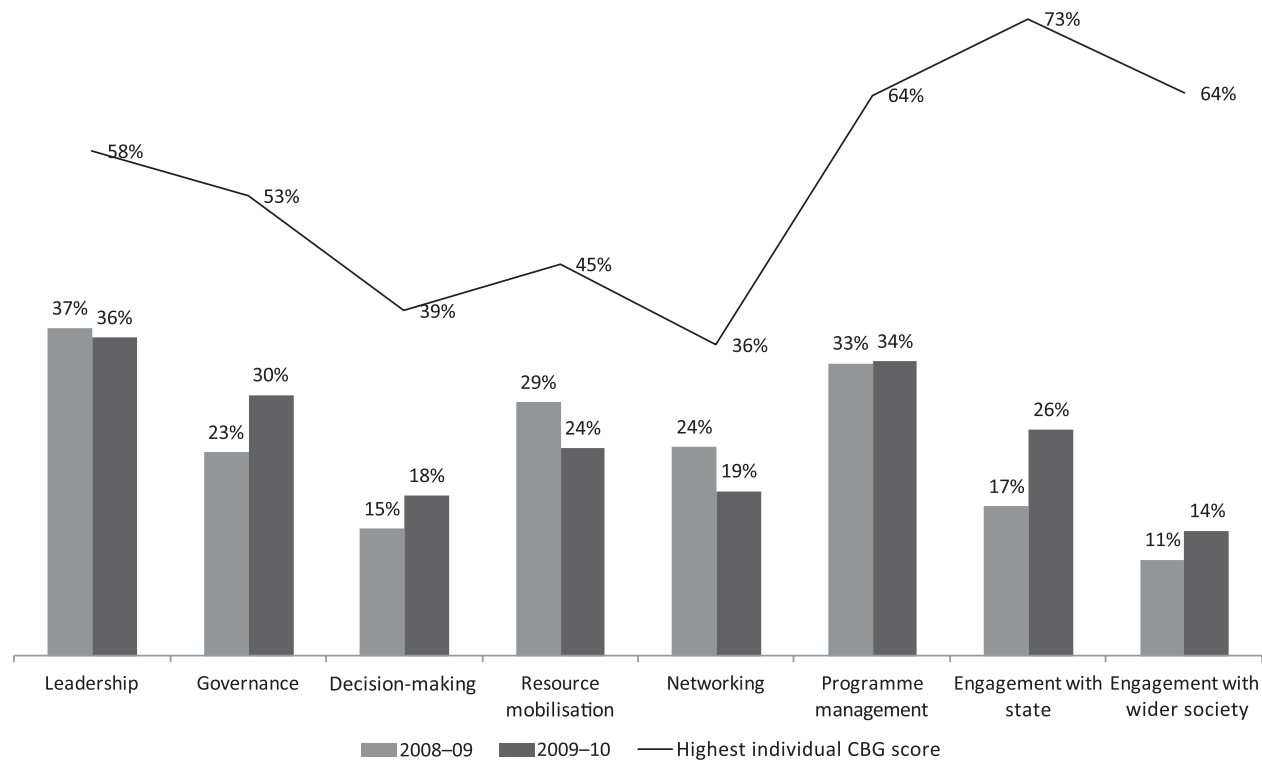

second round of the survey, newer CBGs had surpassed the older ones in Governance.

In general, older and newer $\mathrm{CBGs}$ tended to register an increase or decrease on the same parameter scores between rounds of the survey, the exceptions being Leadership (a slight increase among older CBGs, a slight decrease among newer ones) and Engagement with Wider Society (a large increase among older CBGs, a slight decrease among newer ones). In the parameters of Decision Making and Programme Management, the increase in average score between rounds of the survey was more marked for older CBGs than for newer ones.

\section{Analysis by score on a 7-point scale}

In order to present the progress of each CBG towards transition readiness in a way that could be instantly grasped by its members and which allowed for straightforward comparisons between parameters, Avahan devised a simple scale dividing the possible range of percentage scores into seven equal parts (table 3). Avahan's goal for community mobilisation was that by 2013, most CBGs would score at least 5 on the 7-point scale (equivalent to at least $57 \%$ on each parameter).
Figure 5 shows the distribution of the $25 \mathrm{CBGs}$ along the 7 -point scale for each of the parameters in the second monitoring round. In 2009-2010, there were four parameters on which some of the CBGs (ranging from one to three) were considered transition-ready: Leadership, Programme Management, Engagement with the State and Engagement with Wider Society. On three parameters-Decision Making, Networking and Engagement with Wider Society-between 18 and 23 of the CBGs scored 1 or 2 on the scale.

\section{DISCUSSION}

The systematic monitoring and measurement of community organisational development (or ownership and participation) in the Avahan programme took place to guide the programme, using the same standards across a wide range of CBGs in six states in India. The concepts underlying much of what we accept as operational standards in community development, approaches for empowering communities and strengthening their capacity, remain disputed, and measuring progress is therefore difficult. ${ }^{23}$ The COPI defined these concepts and developed numeric scoring for comparison, but with the purpose

Figure 3 Median and highest parameter scores of 25 communitybased groups, disaggregated by highrisk group type, in two rounds of monitoring with Community Ownership and Preparedness Index (COPI) tool.

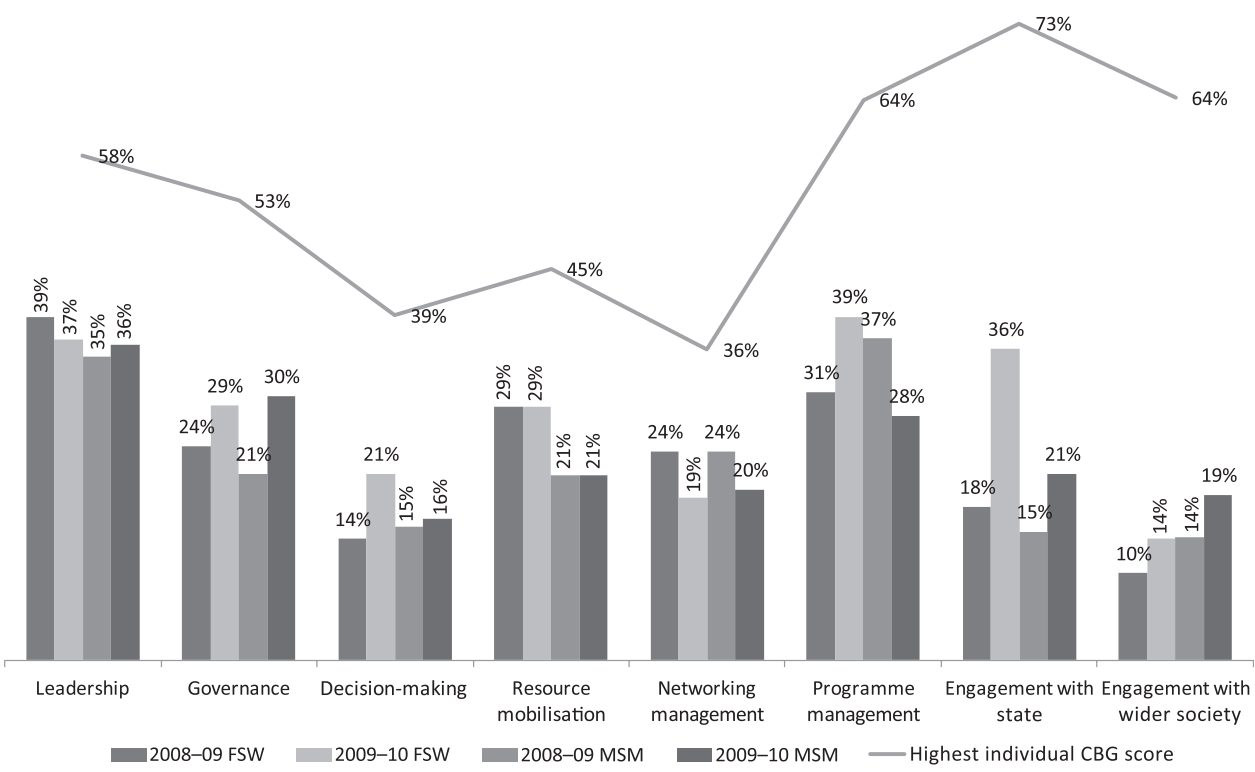


Figure 4 Median and highest parameter scores of 25 communitybased groups, disaggregated by year of foundation, in two rounds of monitoring with Community Ownership and Preparedness Index (COPI) tool.

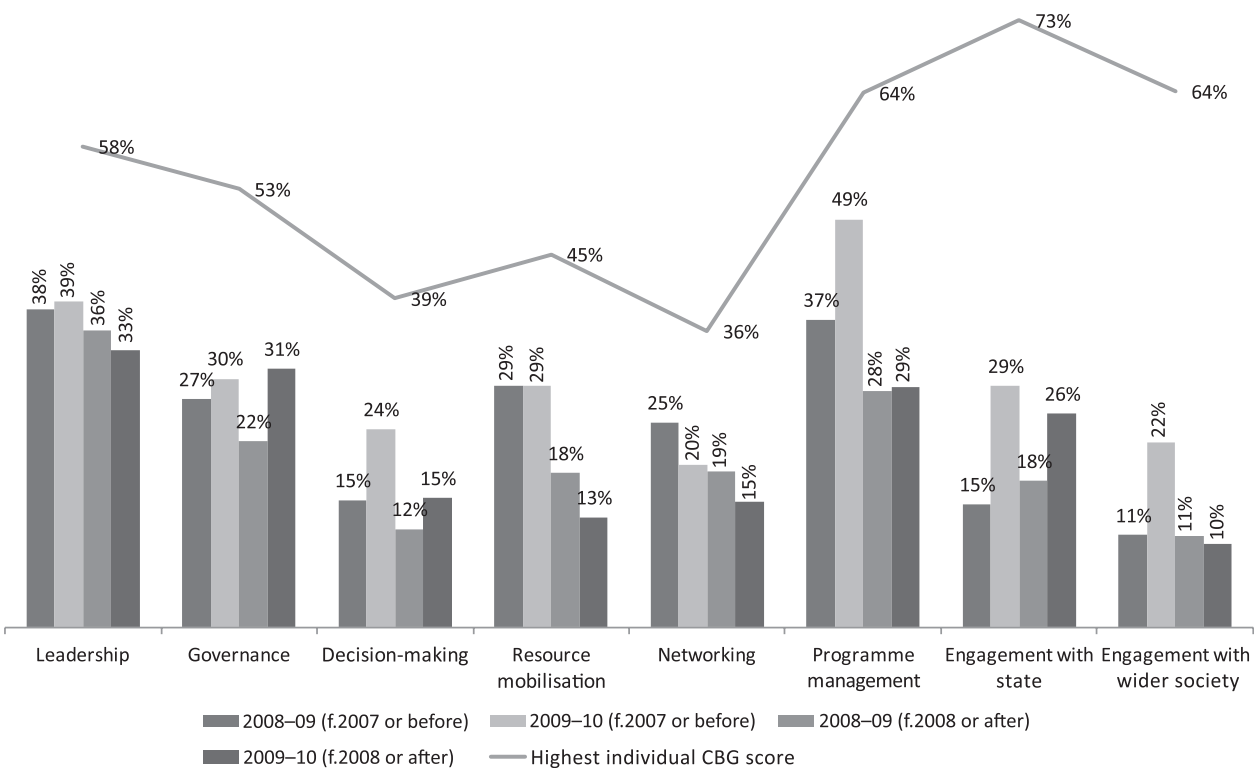

of monitoring progress rather than drawing conclusions through evaluative comparisons. All the variables of a CBG's context and length of existence cannot be adjusted for in an analysis of this nature, and comparisons between community groups from different contexts may often not be valid. ${ }^{24}$

Comparison of the same CBGs over time using a variety of nuanced indicator sets created an understanding at a level of detail that was useful for planning purposes to each CBG's leadership, as well as to NGO programme staff. Indicators were adapted from different disciplines including governance, ${ }^{15}$ community development ${ }^{25}$ and NGO management science, which integrates participatory approaches and organisational learning from the private sector. ${ }^{2627}$ Efforts were also made to measure qualitative, as well as quantitative aspects of programming, drawing from the field of participatory development. ${ }^{14}$ By combining a broad scope with in-depth analysis, the COPI produced results revealing common themes among many of the CBGs surveyed which may be considered indicative of the nature of community ownership and preparedness in the Avahan programme.

\section{Community involvement in project implementation}

Across the CBGs surveyed (and the Avahan programme more broadly), a common feature has been the presence of strong community leaders and their awareness of the processes of service delivery. Empowering community leaders with programme knowledge was a strategy of the Avahan programme that was developed in successive stages: first, peer educators were engaged and cadres of community leaders were trained to play a lead role in forming, as well as strengthening CBGs. ${ }^{28}$ Second, peer educators became involved in almost every component of service delivery and gained a nuanced awareness of project processes. ${ }^{8}$ These efforts are reflected in the relatively high average scores for the parameters of Leadership and Programme Management across both rounds of the monitoring exercise (in 2009-2010, these scores were $36 \%$ and $34 \%$, respectively). This approach is evident in the progress made by FSW CBGs, and in the Round 1 results for HR-MSM CBGs. While there appears to be a levelling-off of leadership progress among older CBGs in the second monitoring round, this does not account for the work done to recruit and train more inexperienced leaders. Taken in this context, the aggregate changes in leadership are dependent upon other factors in the programme and can only be interpreted with an understanding of dynamics on the ground.

\section{Creating institutional spaces for wider community involvement}

The dimension of organisational strength is comprised of the parameters of Leadership, Governance and Decision Making. Although leadership development was the main focus of programme efforts early on, leaders did not necessarily have a strong constituency. Round 1 data showed Leadership to be the highest-scoring among the three parameters in this dimension, but Decision Making was almost the lowest-scoring of all parameters (figure 2). The 1-year period between Rounds 1 and 2 of the monitoring exercise produced marked increases in the scores for Governance and Decision Making for FSW CBGs, ranging from five to seven percentage points, and a similarly marked increase in the Governance score (nine percentage points) for HR-MSM CBGs (figure 3). These data reflect the programmatic focus on strengthening of governance which many CBGs underwent during the year: some became legally registered $\mathrm{CBOs}$, and in some places leadership elections were held. In the state of Andhra Pradesh, site-level systems were established to enable groups to discuss the overall functioning of the CBG's leadership team. Increasingly, decision-making systems were refined, and community members started playing a greater role in the functioning of CBGs. Some of the areas addressed included establishing fixed membership fees, and the selection of CBG staff and CBG representatives to state networks of community groups. In the states of Andhra Pradesh and Tamil Nadu, community members reported that exposure to the COPI helped them understand what they could do to become a strong CBG.

Table 3 Scale for monitoring progress of community-based groups using Community Ownership and Preparedness Index (COPI) tool

\begin{tabular}{llllllll}
\hline Scale & $\mathbf{1}$ & $\mathbf{2}$ & $\mathbf{3}$ & $\mathbf{4}$ & $\mathbf{5}$ & $\mathbf{6}$ & $\mathbf{7}$ \\
\hline Weighted parameter score (all in \%) & $0-14.3$ & $14.4-28.6$ & $28.7-42.9$ & $43.0-57.1$ & $57.2-71.4$ & $71.5-85.7$ & $85.8-100$ \\
\hline
\end{tabular}


Figure 5 Distribution of 25 community-based groups on a 7-point scale, disaggregated by parameter, in second round of monitoring with Community Ownership and Preparedness Index (COPI) tool. The figure in each circle shows the number of CBGs that attained a particular score on the 7-point scale, and the size of the circle represents the proportion of the 25 CBGs with that score. For example, 15 CBGs ( $60 \%$ of them) scored 3 on the leadership parameter.

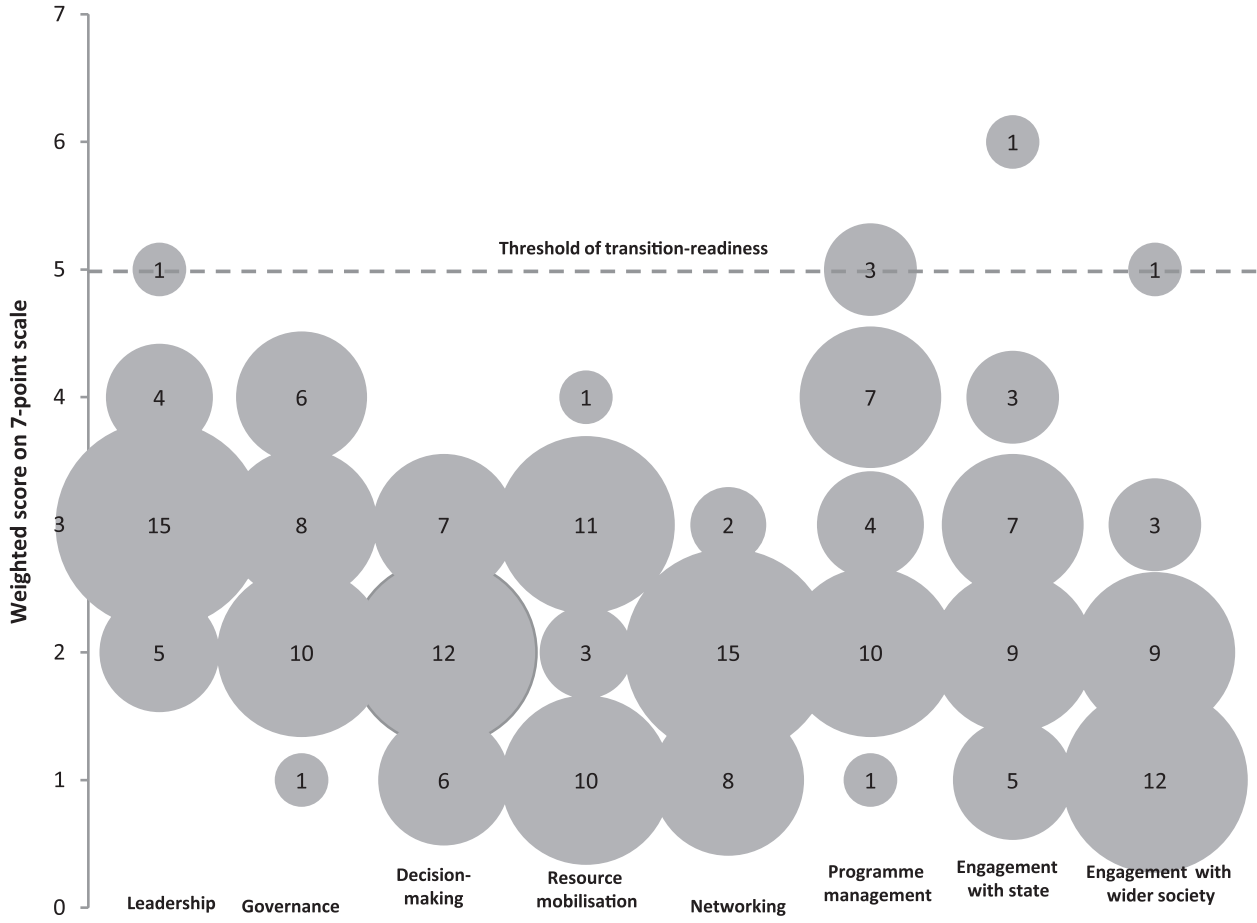

Sustained community responses in India, including the SelfEmployed Women's Association, advocate for a widespread and engaged grassroots base fostered through democratic governance, and shared or democratic decision making in order to sustain movements of the marginalised. ${ }^{29}$ Democratic decision making, far from being merely an idealistic theory advocated by academics, is considered essential as part of strong community organising, not only for the Self-Employed Women's Association but also by the most recognised sex worker collectives in India. ${ }^{130} 31$ While these kinds of issues may be hard to pinpoint, the data from the COPI made it possible to push for changes at the local level, and communities and NGOs were responsive to this. Viewing organisational change as a factor of strong leadership along with good governance and decision making, reflects both the competing and complementary attributes of the COPI parameters.

\section{Mobilising financial resources}

The most challenging area for CBGs across the programme has been mobilisation of financial resources. Groups have relied largely upon their own members to support programme activities by volunteering their time. But apart from receiving financial support from the NGO, most CBGs have raised funds only by levying nominal membership fees. While this indicates a level of commitment among CBG members, it is not sufficient to ensure ongoing financial stability as the amount is less than the financial support offered by implementing NGOs. The COPI investigated the ability of CBGs to develop funding proposals and apply for funding to state bodies and NGOs, but most CBGs were not yet at this stage. Success in resource mobilisation is evident only among a handful of well-networked CBGs that were capable of raising resources by organising cultural events. A further area of future development must also be that of strengthening the management systems of the CBGs, only half of which, for example, had opened bank accounts. It is interesting to note that when the weighting of the parameters in the COPI was first being discussed, communities suggested that the Resource Mobilisation parameter be much more heavily weighted than the others, indicating a strong awareness of what a challenge it would be to procure resources. In practice, however, it may, for now, remain the responsibility of NGOs and donors to ensure that CBGs get the funding they need to survive.

\section{Engagement on issues of rights, entitlements and addressing stigma}

One of the central questions about the long-term viability of the $\mathrm{CBG}$ is: what is the incentive for members to join? In almost all CBGs surveyed, $\mathrm{CBG}$ members reported that they join groups, in large part, to address issues of rights, social entitlements and stigma. While the HIV prevention programme has been central to Avahan's interest, addressing crucial social, economic and political factors has greater daily relevance for high-risk individuals.

CBGs apparently recognised the importance of addressing this priority of their constituents: they both educated communities about their rights, and took action. Data show that the programme has contributed to community awareness of rights and entitlements in more than 10 different areas. ${ }^{31}$ From 2008-2009 to 2009-2010 there was a nine-percentage-point increase in community-led engagement with the state to advocate for civil and political rights and entitlements for their members, the single greatest increase of any parameter between rounds of the survey (among FSW CBGs, the increase was 18 points, and among HR-MSM CBGs it was six points). This substantial increase in $\mathrm{CBG}$ engagement during this period occurred because in many states, CBGs had focused on securing entitlements such as housing and health insurance.

While these figures indicate progress in communities advocating for their rights with institutions that can be held accountable, the greater challenge may be advocacy for their rights in wider society, where changes in norms can be harder to achieve. Over the two rounds of the survey, the CBGs' engagement with larger society increased by four and five percentage points for FSW and HR-MSM CBGs, respectively (figure 3). While older CBGs increased their score on this parameter by $11 \%$ points, on average, younger ones registered a slight decrease 


\section{What is already known on this subject}

- Public health approaches are often designed to increase participation in health interventions, assuming that this will result in an increase in collective agency to support healthseeking behaviours and an enabling environment for disease prevention. HIV prevention programmes for high-risk groups have been among the most documented operational approaches using this kind of model.

- A participatory approach to monitoring in health and development, in which the communities from whom data are collected are involved in analysing those data themselves, can enhance the legitimacy and practical usefulness of such efforts.

- Nevertheless, defining and measuring community mobilisation (and associated terms like collective agency) in large-scale interventions, including for HIV prevention, is problematic.

across the two rounds of the survey (figure 4). This may reflect the reluctance of members of more recently established groups to identify themselves publicly as sex workers or members of the HR-MSM community. Their continued dependence upon NGOs to engage with influential stakeholders, such as religious leaders or the media, demonstrates that they still need others to open doors so that they can try to exercise influence.

The initial monitoring round showed relatively weaker overall CBG development than expected, but despite this, response to the COPI was constructive. NGOs that had previously been suspicious of being scrutinised began to use the analysis for debate, planning and action. This led to changes being made around issues that were once considered difficult to address. Comparison between the rounds of the survey demonstrates that course corrections were made, addressing weaker aspects of CBG development, and leading to further evolution of the groups.

Equipped with detailed information from the COPI about the nature of leadership, internal processes, external associations and their capacity for managing activities, the CBGs are being

\section{What this study adds}

- It is possible to design and implement a monitoring approach that captures both qualitative and quantitative data on processes of community mobilisation, has means of verification and offers reliable data.

- A participatory approach to the design, implementation and analysis of a community monitoring survey is possible and enhances the survey's legitimacy in the eyes of the high-risk community.

- A survey tool designed to offer multiple options for presenting and analysing data can be used for planning purposes not only by programme staff but also by the community-based groups themselves.

- Across six highly varied states in India, programmes were able to learn from local-level monitoring in which high-risk communities themselves reflected on their progress and plans for the future. This accelerated the maturing of communitybased groups, suggesting greater potential for their long-term sustainability.

\section{Policy implications}

In a global environment where resources for health are increasingly tight, methods that can improve programme performance are essential. The COPI functions to continually improve programme design and implementation by identifying strengths and weaknesses in progress towards targets for community organisational readiness. The method may contribute to strengthening programmatic approaches and the commitment to undertaking such approaches in policy making.

strengthened as platforms for fostering accountable and appropriate clinical and social responses to HIV in India. Their evolution is being determined through participatory planning, where the goal is to enable communities themselves to plan and lead their own development. Across six highly varied states in India, programmes have been able to learn from local-level monitoring in which high-risk communities themselves reflect on their progress and plans for the future. This has accelerated the maturing of CBGs, suggesting greater potential for their long-term sustainability. This monitoring exercise will continue through 2015. The tools will not only help CBGs to monitor their progress, but also facilitate annual planning processes in terms of setting institutional priorities.

Acknowledgements The authors would like to acknowledge additional members of the COPI design team: Joseph M J, Anindo Banerjee, Nalini Jamela (FSW) and Priya Babu (TG). The team would also like to thank Tisha Wheeler, Usha Kiran, James Moore and Gina Dallabetta of the Bill \& Melinda Gates Foundation for working with it to develop a monitoring instrument that met the needs of the programme and Rick Davies and Geeta K (expert on gender, sexuality and violence) for their technical input. The tools were designed after consultation among community groups in the Districts of Kolhapur in Maharashtra, Chittoor, Nellore in Andhra Pradesh, Ramanagaram in Karnataka and Imphal in Manipur. We are grateful for the cooperation of Avahan's lead implementing partners in the implementation of both rounds of the monitoring tool: Alliance for AIDS Action Project (India HIV/AIDS Alliance, Andhra Pradesh), Swagati and Nestam Projects (Hindustan Latex Family Planning Promotion Trust, Andhra Pradesh), Corridors and Project Sankalp (Karnataka Health Promotion Trust, Karnataka), Aastha Project (Family Health International, Maharashtra), Mukta Project (Pathfinder International, Maharashtra), Project ORCHID (Emmanuel Hospital Association, Manipur and Nagaland) and Tamil Nadu AIDS Initiative (Voluntary Health Services, Tamil Nadu). Above all, we thank all the community-based groups that participated in the survey. This research was funded by the Bill \& Melinda Gates Foundation. The views expressed herein are those of the authors and do not necessarily reflect the official policy or position of the Bill \& Melinda Gates Foundation.

Contributors All authors named in this manuscript have met the following criteria for involvement: (1) conception and design, acquisition of data or analysis and interpretation of data; (2) drafting the article or revising it critically for important intellectual content; (3) final approval of the version published. Each author has participated sufficiently in the work to take public responsibility for appropriate portions of the content. There is no one else who fulfils the criteria who has not been included as an author. I certify that neither this manuscript nor one with substantially similar content under my authorship has been published, or is being considered for publication elsewhere. I have access to all data upon which the manuscript is based and will provide such data upon request to the editors or their assignees. According to the definition given by the International Committee of Medical Journal Editors (ICMJE), I qualify for authorship based on making one or more of the substantial contributions to the intellectual content: conception and design, acquisition of data, and/or analysis and interpretation of data. Furthermore, I have participated in drafting of the manuscript and/or critical revision of the manuscript for important intellectual content.

Funding This work was supported by the Bill \& Melinda Gates Foundation under the HIV/AIDS Division through a range of grants in the Avahan portfolio, including grants of the following identification numbers: 29868, 29928, 30080, 30111, 30121, 30138, 30148, 30177, 30183, 30553, 3128039418 , and 5189. 
Provenance and peer review Commissioned; externally peer reviewed.

Data sharing statement All data is open access.

\section{REFERENCES}

1. Jana S, Basu I, Rotheram-Borus MJ, et al. The Sonagachi Project: a sustainable community intervention program. AIDS Educ Prev 2004;16:405-14.

2. Chattopadhyay A, McKaig RG. Social development of commercial sex workers in India: an essential step in HIV/AIDS prevention. AIDS Patient Care ST 2004;18:159-68.

3. Boyce B. Evaluation of the Effects and Effectiveness of InterAct IPC Work with Truck Drivers and Intravenous Drug Users, India. London: Thomas Coram Research Unit, Institute of Education, University of London, 2008

4. Biradavolu MR, Burris S, George A, et al. Can sex workers regulate police? Learning from an HIV prevention project for sex workers in southern India. Soc Sci Med 2009;68:1541-7.

5. Sarkar S. Community engagement in HIV prevention in Asia: going from 'for the community' to 'by the community' - must we wait for more evidence? Sex Transmit Infect 2010;86(Suppl 1):i2.

6. Blankenship KM, West BS, Kershaw TS, et al. Power, community mobilization, and condom use practices among female sex workers in Andhra Pradesh, India. AIDS 2008;22(Suppl 5):S109-16.

7. Chambers R. Whose Reality Counts? Putting the First Last. London: Intermediate Technology Publications, 1997.

8. Wheeler T, Kiran U, Dallabetta G, et al. Learning about scale, measurement and community mobilisation: reflections on the implementation of the Avahan HIV/AIDS initiative in India. J Epidemiol Community Health 2012;66:ii16-ii25.

9. Avahan-The India AIDS Initiative. The Business of HIV Prevention at Scale. New Delhi: Bill \& Melinda Gates Foundation, 2008

10. Thomas T, Narayanan P, Wheeler T, et al. Design of a Community Ownership and Preparedness Index: using data to inform the capacity development of communitybased groups. J Epidemiol Community Health 2012;66:ii26-ii33.

11. Reza-Paul S, Lorway R, O'Brien N, et al. Sex worker-led structural interventions in India: a case study on addressing violence in HIV prevention through the Ashodaya Samithi collective in Mysore. Indian J Med Res 2012;135:98-106.

12. The Power to Tackle Violence: Avahan's Experience with Community Led Crisis Response in India. New Delhi: Bill \& Melinda Gates Foundation, 2009. http://www. gatesfoundation.org/avahan/Documents/Avahan_PowerToTackleViolence.pdf

13. Bennett S, Singh S, Ozawa S, et al. Sustainability of donor programs: evaluating and informing the transition of a large HIV prevention program in India to local ownership. Glob Health Action 2011;4:7360.

14. Estrella M, Gaventa J. Who Counts Reality? Participatory Monitoring and Evaluation: A Literature Review. Brighton, UK: Institute of Development Studies, 1998.
15. Gaventa J, Pettit J, Cornish L. Powercube: Understanding Power for Social Change. Brighton, UK: Institute of Development Studies, 2011. http://www.powercube.net/

16. Campbell C, Mzaidume Z. Grassroots participation, peer education, and HIV prevention by sex workers in South Africa. Am J Public Health 2001;91:1978-86.

17. Gaventa J. Finding the spaces for change: a power analysis. Brighton, UK. IDS Bull 2006;37:23-33.

18. Bebbington A, Hickey S, Mitlin DC. Can NGOs Make a Difference? The Challenge of Development Alternatives. London: Zed Books, 2007.

19. Deininger K, Liu Y. Longer-term Economic Impacts of Self-Help Groups in India. World Bank Policy Research Working Paper No. 4886. Washington, DC: World Bank 2009.

20. Saidel T, Adhikary R, Mainkar M, et al. Baseline integrated behavioural and biologica assessment among most at-risk populations in six high-prevalence states of India: design and implementation challenges. AIDS 2008:22(Suppl 5):S17-34.

21. Chandrasekaran P, Dallabetta G, Loo V, et al. Evaluation design for large-scale HIV prevention programmes: the case of Avahan, the India AIDS initiative. AIDS 2008:22 (Suppl 5):S1-15

22. http://www. filemaker.com/

23. White S. Depoliticising development: the uses and abuses of participation. Dev Pract 1996;6:6-15

24. Campos J, Coupal FP. Participatory Evaluation: a Handbook for UNDP Staff. New York: United Nations Development Programme, Strategic Planning and Evaluation Unit, 1996

25. Pearce J, ed. Development, NGOs and Civil Society. UK: Oxfam, 2000.

26. Simbi M, Thom G. "Implementation by proxy": the next step in power relationships between Northern and Southern NGOs? In: Lewis D, Wallace T, eds. New Rules and Relevance: Development NGOs and the Challenge of Change. West Hartford, USA: Kumarian Press, 2000

27. James R. Demystifying Organisation Development: Practical Capacity-building Experiences of African NGOs. Oxford: International Training and NGO Research Centre, 1998

28. Managing HIV Prevention from the Ground up: Avahan's Experience with Peer Led Outreach at Scale in India. New Delhi: Bill \& Melinda Gates Foundation, 2009. http:// www.gatesfoundation.org/avahan/Documents/Avahan_PeerOutreach.pdf

29. Rose K. Where Women are Leaders: The SEWA Movement in India. London: Zed Books, 1992.

30. Pillai S, Seshu M, Shivdas M. Embracing the rights of people in prostitution and sex workers, to address HIV and AIDS effectively. Gend Dev 2008;16:313-26.

31. Narayanan P, Moulasha K, Wheeler $T$, et al. Using data to understand programmatic shifts in the Avahan HIV prevention program at the community level. In: Evaluation of Community Response to HIVIAIDS. Washington, DC: World Bank, 2012. Publication pending. 